\title{
A Quartic Conformally Covariant Differential Operator for Arbitrary Pseudo-Riemannian Manifolds (Summary) ${ }^{\star}$
}

Stephen M. PANEITZ

\section{Deceased}

Received March 27, 2008 from Michael Eastwood; Published online March 30, 2008

Original article is available at http://www.emis.de/journals/SIGMA/2008/036/

\begin{abstract}
This is the original manuscript dated March $9^{\text {th }}$ 1983, typeset by the Editors for the Proceedings of the Midwest Geometry Conference 2007 held in memory of Thomas Branson. Stephen Paneitz passed away on September $1^{\text {st }} 1983$ while attending a conference in Clausthal and the manuscript was never published. For more than 20 years these few pages were circulated informally. In November 2004, as a service to the mathematical community, Tom Branson added a scan of the manuscript to his website. Here we make it available more formally. It is surely one of the most cited unpublished articles. The differential operator defined in this article plays a key rôle in conformal differential geometry in dimension 4 and is now known as the Paneitz operator.
\end{abstract}

Key words: Paneitz operator; conformal covariance

2000 Mathematics Subject Classification: 53A30; 58J70

Let $M^{\prime}$ be a pseudo-Riemannian manifold of dimension $n>1$. The fourth order operator defined below (equation (3)) bears some analogy with the second-order shift of the LaplaceBeltrami operator $\mathcal{L}$ by a multiple of the scalar curvature $R$, namely

$$
\mathcal{L}+\frac{n-2}{4(n-1)} R
$$

defined by Ørsted, and studied by others in special cases. The relation satisfied by operators of the form (1) is the following: if $M$ is a manifold of dimension $n>1$ and $g_{1}$ and $g_{2}$ are two metric tensors on $M$ that satisfy $g_{1}=p^{2} g_{2}$ for some positive function $p$, then

$$
p^{(n+2) / 2}\left(\mathcal{L}_{g_{1}}+\frac{n-2}{4(n-1)} R_{g_{1}}\right) \phi=\left(\mathcal{L}_{g_{2}}+\frac{n-2}{4(n-1)} R_{g_{2}}\right)\left(p^{(n-2) / 2} \phi\right)
$$

for all smooth functions $\phi$, where $\mathcal{L}_{g_{i}}$ and $R_{g_{i}}$ are the Laplace-Beltrami operator and scalar curvature determined by the pseudo-Riemannian metric $g_{i}$, for $i=1,2$. It is not necessary that the scalar curvatures be constants. We will refer to the multipliers $p^{(n-2) / 2}$ and $p^{(n+2) / 2}$ as the "initial" and "final" multipliers, respectively.

Note that when $n=2$ (and only in this case), the initial multiplier is 1 , the multiplicative part of the operator (1) is 0 , the equation $\mathcal{L}_{g} \phi=0$ is independent of the metric $g$, and thus these solutions are simply related, modulo topology, to the harmonic 1-forms $\omega$ satisfying $d \omega=\delta \omega=0$, merely by exterior differentiation.

The quartic operator defined below has an entirely comparable list of special features in the physical case of dimension $n=4$. Its solutions in this case are related to the conformally invariant class of harmonic differential forms, which being forms of half the dimension of the manifold, are the two-forms; if the metrics $g$ have Lorentzian signature the equations of harmonicity are the Maxwell equations.

${ }^{\star}$ This paper is a contribution to the Proceedings of the 2007 Midwest Geometry Conference in honor of Thomas P. Branson. The full collection is available at http://www.emis.de/journals/SIGMA/MGC2007.html 
It seems reasonable to conjecture the existence of analogous operators of arbitrary higher even order; indeed, powers of the flat wave operator in Minkowski space were shown to have a conformal covariance property in Jakobsen's thesis and work of Jakobsen-Vergne.

To define the quartic operator, we must assume that the dimension of the manifold is neither 1 nor 2. Given a pseudo-Riemannian metric $g$ on $M$, with associated Laplace-Beltrami operator $\mathcal{L}_{g}$, scalar curvature $R$, Ricci tensor components $R_{i j}$ (tensorial notation referring to coordinates $\left.x^{1}, x^{2}, \ldots, x^{n}\right)$, metric components $g_{i j}$ and covariant derivatives $T_{i}=\nabla_{\frac{\partial}{\partial x^{i}}}$, define

$$
\begin{aligned}
\mathcal{Q}(g) \phi= & \left(\mathcal{L}_{g}\right)^{2} \phi+T_{j}\left[\left(-\frac{4}{n-2} R^{i j}+\frac{n^{2}-4 n+8}{2(n-1)(n-2)} g^{i j} R\right) \frac{\partial}{\partial x^{i}} \phi\right] \\
& +\left[\frac{n-4}{4(n-1)}\left(\mathcal{L}_{g} R\right)-\frac{n-4}{(n-2)^{2}} R^{i j} R_{i j}+\frac{(n-4)\left(n^{3}-4 n^{2}+16 n-16\right)}{16(n-1)^{2}(n-2)^{2}} R^{2}\right] \phi
\end{aligned}
$$

for scalar functions $\phi$ (the usual summation convention being followed).

Theorem 1. Let $M$ be an arbitrary smooth manifold of dimension $n>2$, and assume given two pseudo-Riemannian metrics $g_{1}$ and $g_{2}$ on $M$ related by $g_{1}=p^{2} g_{2}$ for a positive function $p$. Then for all scalar functions $\phi$,

$$
p^{(n+4) / 2} \mathcal{Q}\left(g_{1}\right) \phi=\mathcal{Q}\left(g_{2}\right)\left(p^{(n-4) / 2} \phi\right) .
$$

When $n=4$,

$$
\mathcal{Q}(g) \phi=\mathcal{L}_{g}{ }^{2} \phi-2 T_{j}\left[\left(R^{i j}-\frac{1}{3} g^{i j} R\right) \frac{\partial}{\partial x^{i}} \phi\right],
$$

and the kernel of this operator is independent of the metric $g$, and also invariant under the ordinary pointwise action of the conformal group $\left(M, g_{1}\right)$ (or equivalently $\left(M, g_{2}\right)$ ) on functions.

In the special case of a conformally flat four-dimensional manifold $M$ of Lorentzian signature, we can sketch the relation to Maxwell's equations alluded to above. For such an $M$, any smooth local conformal transformation extends uniquely to $\widetilde{M}=\mathbb{R} \times S^{3}$. But now any global distribution two-form solution of Maxwell's equations on $\widetilde{M}$ is automatically invariant under the central transformation of $\widetilde{M}$ sending $(t, p)$ into $(t+\pi$, antipodal point to $p)$, as shown in the work of I.E. Segal. The associated compact quotient manifold of $\widetilde{M}$, denoted $\bar{M}$, is casually identifiable with the conformal compactification of four-dimensional Minkowski space. Now set $g_{c}=d t^{2}-d s^{2}$ (the Einstein metric on $\mathbb{R} \times S^{3}$ ), and set $X_{0}=\partial / \partial t$ and $\Delta$ the Laplacian on $S^{3}$. Then

$$
\mathcal{Q}\left(g_{c}\right)=\left(X_{0}^{2}-\Delta\right)^{2}+4 X_{0}^{2},
$$

and

$$
p^{4} \mathcal{Q}\left(g_{c}\right)=\mathcal{Q}\left(g_{f}\right)=\left(\mathcal{L}_{g_{f}}\right)^{2},
$$

where $g_{f}=\left(d x^{0}\right)^{2}-\left(d x^{1}\right)^{2}-\left(d x^{2}\right)^{2}-\left(d x^{3}\right)^{2}$ is the flat metric, and we have $p^{2} g_{f}=g_{c}$. (Regarding $S^{3}$ as $\mathrm{SU}(2)$, one can take $p(t, u)=\frac{1}{2} \cos t+\frac{1}{4} \operatorname{tr}(u)(t \in \mathbb{R}, u \in \mathrm{SU}(2))$.)

Theorem 2. $\mathcal{Q}\left(g_{c}\right) \phi=0$ has an infinite-dimensional space of smooth solutions on $\bar{M}$ (all solutions on $\widetilde{M}$ are lifted up from $\bar{M}$ ), and this equation satisfies Huygens' principle. This space of solutions is the unique conformally invariant closed subspace of the space of all smooth fields on $\bar{M}$ whose elements are determined by Cauchy data of a finite number of $X_{0}$-derivatives on a space-like surface. 
Any two-form solution of Maxwell's equations on $\bar{M}$ is the exterior derivative of a 1-form $A$ on $\bar{M}$ satisfying $d *_{c} A=0$ and $A\left(X_{0}\right)=0$ (gauge conditions; $*_{c}$ is the $*$-operator determined by $g_{c}$ ). The space of 1 -forms $A$ on $\bar{M}$ satisfying $d *_{c} d A=0$ (Maxwell's equations) and $d *_{c} A=0$ and $A\left(X_{0}\right)=0$ is not conformally invariant, but its direct sum with the conformally invariant space of exterior derivatives of functions $\phi$ satisfying $\mathcal{Q}\left(g_{c}\right) \phi=0$ (or this space augmented by $d t$, namely the corresponding space of closed 1-forms that are locally exterior derivatives of functions $\phi$ satisfying $\mathcal{Q}\left(g_{c}\right) \phi=0$ ), is conformally invariant.

Moreover, all the conformally invariant closed subspaces of the real smooth 1 -forms on $\bar{M}$ satisfying Maxwell's equations are generated by the above-noted conformally invariant subspaces and the obvious one of all closed 1-forms on $\bar{M}$. 